\title{
Quantitative Analysis of Myelin sheaths in a Mouse Model of Experimental Autoimmune Encephalitis
}

\author{
Vered Behar ${ }^{*}$, Miri Horowitz ${ }^{*}$, Alon Sabban ${ }^{*}$, Adi Mayk ${ }^{* *}$, Debbie Jodorkovsky ${ }^{*}$, Eran \\ Blaugrund $^{* *}$, and Abraham Nyska ${ }^{* * *}$. \\ * Quantomix, Ltd., P.O. Box 4037, Ness Ziona, Israel. \\ ** Teva, Ltd., P.O. Box 8077, Sapir Industrial Zone, Netanya, Israel. \\ *** Laboratory of Experimental Pathology, National Institute of Environmental Health Sciences \\ (NIEHS), National Institutes of Health, Research Triangle Park, NC, United States.
}

Multiple sclerosis (MS) is an immune-mediated inflammatory demyelinating disease of the central nervous system (CNS) for which the etiology is unknown. Pathological and immunological aspects of MS may be studied in the experimental allergic encephalomyelitis (EAE) model, induced in mice by immunization with spinal cord homogenate or CNS myelin components. Clinical neurological scoring is the generally utilized assessment method, but there is a need for a rapid and high-resolution microscopic evaluation of myelin or axonal damage. We investigated the utility of a newly developed technology of scanning electron microscopy (SEM) designed for analysis of fully wet tissue specimens (wet SEM) [1]. The WETSEM ${ }^{\mathrm{TM}}$ technology is based on a sample holder that isolates thick tissue samples (up to $1.5 \mathrm{~mm}$ ) from the vacuum in the electron microscope's chamber. Using only minimal preparation steps, the sample is placed in the holder and scanned by the electron beam to obtain high-resolution images based on differences in the atomic composition within the sample. We compared control with sick EAE animals by conventional histology and the wet SEM technology. We then applied custom-made image analysis software for the quantitative analysis of the myelin sheaths. Our investigations 
indicate that the wet SEM technology rendered myelin sheaths, axons, and inflammatory cells simple to differentiate. We conclude that the wet SEM technology provides a quick, accurate, and detailed structural evaluation of the spinal cord and can be applied as a routine test for potential new MS therapies.

[1] Thiberge et al. PNAS 101:3346 (2004) 